\title{
Probabilistic models for the extent of damage in degrading reinforced concrete structures
}

\author{
B. Sudret \\ Electricité de France, R\&D Division, Site des Renardières \\ F-77818 Moret-sur-Loing, FRANCE
}

\begin{abstract}
Describing accurately damage in degrading reinforced concrete structures is of major interest in the context of durability analysis and maintenance. Due to numerous sources of uncertainty in the degradation models, a probabilistic approach is suitable. The probabilistic description of the extent of damage requires introducing random fields for modelling the spatial variability of the various parameters. In this paper, a general formulation for the spatial extent of damage is set up. This formulation allows to derive closed-form expressions for the mean value and standard deviation of the latter. Accordingly, practical computations can be carried out without discretizing the input fields. In order to check the accuracy of the proposed implementation, Monte Carlo simulation (MCS) of the extent of damage is also carried out, using an efficient random field discretization technique known as EOLE. Both approaches are compared to study the extent of rebars corrosion in a RC beam subjected to concrete carbonation. Furthermore, the Monte Carlo approach allows to compute the full probabilistic content on the extent of damage, e.g. histograms. It was shown that these histograms have a non trivial shape, in the sense that probability spikes exist for the bound values (case of undamaged and fully damaged structures). The influence of the auto-correlation function of the various input random fields and that of their scale of fluctuation is finally studied.
\end{abstract}

Keywords : extent of damage / space-variant reliability / time-variant reliability / degradation models / concrete carbonation / rebars corrosion / random fields / EOLE method /

\section{Introduction}

Probabilistic models of concrete degradation have been intensively studied in the past ten years. The most important degradation mechanism considered in the literature is the corrosion of the rebars due to chloride ingress in the concrete mass or concrete carbonation. This mechanism is of utmost importance in the ageing of bridge structures that are submitted to deicing salts, or any structure in a marine environment $[1,2,3,4]$. Authors have focused on the prediction of the initiation time for corrosion and / or the estimation of the residual strength of structures.

Recent advances in this field have pointed out the necessity of modelling the spatial variability of the model parameters in order to be able to characterize, not only the probability of degradation, but 
also the extent of damage $[5,6,7]$. This extent of damage is the natural variable that characterizes the global state of damage of the structure, and that may be used in optimizing maintenance policies $[5,8,9,10]$.

In this paper, a general formulation for spatially variable degradation models is proposed. The socalled point-in-space and space-variant reliability problems are recalled in Section 2 [11]. Then the extent of damage is given a proper definition, from which analytical derivations are carried out in order to compute the first two statistical moments (Section 3). Efficient implementations of these formulæ (based on the First Order Reliability Method (FORM) and Monte Carlo simulation) are proposed in Section 4.

In order to evaluate the accuracy of the analytical approach, an alternative framework for the direct estimation of the extent of damage by Monte Carlo simulation is proposed. This requires the use of random field discretization techniques and the post-processing of the simulation results. Both approaches (called "analytical" and "field discretization" in the sequel) are compared on an application example, which considers carbonation-induced corrosion. Finally, the results of Monte Carlo simulation allow to plot histograms of the extent of damage, whose specific shape is studied.

\section{Spatially varying probabilistic degradation models}

\subsection{A class of degradation models}

The degradation of structures in time may be defined in a broad sense as the loss of certain properties as the result of chemical, physical or mechanical processes, or combinations thereof. Concrete structures are submitted to many degradation mechanisms, including rebars corrosion due to chloride ingress or concrete carbonation.

The deterministic models for these degradation mechanisms are usually based on semi-empirical equations that yield a so-called damage measure $D$ (considered here as a scalar quantity) as a function of parameters $z$ and time:

$$
D(t)=\mathcal{M}(\boldsymbol{z}, t)
$$

Examples of damages measures are:

- crack width, which may be modelled as a function of the corrosion rate of the rebars, the concrete cover, the rebars diameter, etc. [5],

- loss of rebars diameter, which depends on the corrosion rate and the time for initiation of corrosion, the latter being modelled specifically in case of chloride or carbonation-induced corrosion [12]

- fatigue damage due to repeated application of stress cycles onto the structure [13].

In order to assess the durability of the structure with respect to a given category of damage, a limit value $\bar{D}$ is usually prescribed (e.g. maximal acceptable crack width, etc.). Note that the damage measure in Eq.(1) is an increasing function of time. Indeed, the degradation phenomena considered in this paper are supposed to be irreversible. 


\subsection{Local reliability problem}

The model parameters in Eq.(1) are in practice uncertain and should be modelled by random variables with prescribed joint probability density function. In this case, the damage measure becomes random. Assessing the state of the structure becomes a reliability problem.

Let us denote by $\boldsymbol{Z}=\left\{Z_{1}, Z_{2}, \ldots, Z_{M}\right\}$ the set of random variables describing the randomness in the degradation model. The failure criterion under consideration is mathematically represented by a limit state function $g(\boldsymbol{Z}, t)$ defined in the space of parameters at time instant $t \in[0, T]$ in a way such that:

- $g(\boldsymbol{Z}, t)>0$ defines the safe state;

- $g(\boldsymbol{Z}, t) \leq 0$ defines the failure state;

- $g(\boldsymbol{Z}, t)=0$ defines the limit state surface.

Denoting by $f_{\boldsymbol{Z}}(\boldsymbol{z})$ the joint probability density function of random vector $\boldsymbol{Z}$, the time-dependent probability of failure of the structure reads:

$$
P_{f}(t)=\int_{g(\boldsymbol{z}, t) \leq 0} f_{\boldsymbol{Z}}(\boldsymbol{z}) d \boldsymbol{z}
$$

or equivalently:

$$
P_{f}(t)=\int_{\mathbb{R}^{M}} \mathbf{1}_{\{g(\boldsymbol{z}, t) \leq 0\}}(\boldsymbol{z}) f_{\boldsymbol{Z}}(\boldsymbol{z}) d \boldsymbol{z}=\mathrm{E}\left[\mathbf{1}_{\{g(\boldsymbol{z}, t) \leq 0\}}(\boldsymbol{z})\right]
$$

where $\mathbf{1}_{\{g(\boldsymbol{z}, t) \leq 0\}}(\boldsymbol{z})$ is the indicator function of the failure domain in the space of parameters.

In the context of degradation models, the limit state function shall be referred to as the damage criterion in the sequel. As an example, if it is defined by a fixed threshold $\bar{D}$ on the damage measure, the limit state function may be of the form:

$$
g(\boldsymbol{Z}, t)=\bar{D}-D(t)=\bar{D}-\mathcal{M}(\boldsymbol{Z}, t)
$$

We further suppose that the input parameters $Z$ are not time-dependent, i.e. that they modelled by random variables and not by random processes. As a consequence, the reliability problem (2), which appears time-dependent, is actually equivalent to a series of time-invariant problems, where $t$ is a dummy parameter. Indeed, due to the above assumption and the fact that the damage measure is increasing in time, any trajectory $g\left(\boldsymbol{z}_{0}, t\right)$ computed from a realization $\boldsymbol{z}_{0}$ of the input random vector is monotonically decreasing with time. The time-dependent aspect of the problem over a time interval $[0, \tau]$ is taken care of by solving the time-invariant problem at the final instant $\tau$. Note that the latter assertion may not hold anymore if some input parameters (e.g. environmental conditions) were modelled by random processes.

In all but academic cases, the integral in Eq.(2) cannot be computed analytically. Thus numerical methods have to be employed. Monte Carlo simulation (MCS) is a versatile tool that takes advantage of the formulation in Eq.(3), where the probability of failure is viewed as the expectation of a function of the parameters. A sample set of input parameters $\left\{\boldsymbol{z}_{1}, \ldots, \boldsymbol{z}_{N_{\text {Sim }}}\right\}$ is generated according to $\boldsymbol{f}_{\boldsymbol{Z}}(\boldsymbol{z})$ and the empirical mean of $\mathbf{1}_{\{g(\boldsymbol{z}, t) \leq 0\}}\left(\boldsymbol{z}_{j}\right)$ is computed. This approach allows to derive confidence intervals for the probability of failure.

The main drawback of Monte Carlo simulation is that the number of samples $N_{\text {Sim }}$ required for a fair prediction becomes untractable when the probability of failure is small. Thus approximate methods such as FORM/SORM have been developed, see e.g. [14] for a detailed presentation. 


\subsection{Space-variant reliability problems}

The above probabilistic degradation model is referred to as zero-dimensional, in the sense that it does not involve any spatial coordinate system attached to the structure. It thus implicitely assumes a complete homogeneity of the degradation all over the structure. In other words, for a given realization $z_{0}$ of the input random vector, the full structure will be either in the safe state (undamaged) or in the failure state (fully damaged). This is of course a coarse simplification of the real world. Moreover, this does not allow to characterize the extent of damage. To address this issue, additional notation shall be introduced.

Suppose that the structure under consideration occupies a volume $\mathcal{D} \subset \mathbb{R}^{d}$, where $d=1,2$ or 3 . The case $d=1$ corresponds to modelling beam or arch structures, the case $d=2$ to plate or shell structures. In order to address the problem of spatial variability, the input random vector in Eq.(2) should be replaced by $M$ multivariate scalar random fields gathered in a vector $\boldsymbol{Z}(\boldsymbol{x})$, where $\boldsymbol{x} \in \mathcal{D}$ is the spatial coordinate. The probabilistic description of these fields is yet to be specified. Note that in practice the following assumptions usually apply:

- the spatial variability of certain components of $Z$ is negligible. They are accordingly modelled as random variables. As a consequence, only a small number of scalar random fields have to be specified in practice. However, for the sake of simplicity, the most general notation $\boldsymbol{Z}(\boldsymbol{x})$ is kept in this section.

- the random field components are homogeneous fields. This is due to the fact that the size of the structure is usually small compared to the scale of fluctuation of the parameters driving the degradation (e.g. environmental parameters such as surface chloride or carbon dioxyde concentration, etc.).

In the space-variant context, the limit state function in Eq.(4) should be replaced by:

$$
g(\boldsymbol{Z}(\boldsymbol{x}), t)=\bar{D}-\mathcal{M}(\boldsymbol{Z}(\boldsymbol{x}), t)
$$

The point-in-space probability of failure is defined in each $\boldsymbol{x} \in \mathbb{R}^{d}$ as follows:

$$
P_{f}(\boldsymbol{x}, t)=\int_{g(\boldsymbol{Z}(\boldsymbol{x}), t) \leq 0} f_{\boldsymbol{Z}(\boldsymbol{x})}(\boldsymbol{z}) d \boldsymbol{z}=\mathrm{E}\left[\mathbf{1}_{\{g(\boldsymbol{Z}(\boldsymbol{x}), t) \leq 0\}}(\boldsymbol{z})\right]
$$

It is computed by freezing $\boldsymbol{x}$ (i.e. replacing the random field $\boldsymbol{Z}(\boldsymbol{x})$ by the corresponding random vector) and $t$, and by applying standard time-invariant reliability methods (MCS, FORM/SORM, etc.). Note that if the random field $\boldsymbol{Z}(\boldsymbol{x})$ is homogeneous, then the same reliability problem is posed at whatever the position of the point $\boldsymbol{x}$ under consideration. Thus the point-in-space probability of failure is independent of $\boldsymbol{x}$ in this case.

The space-variant probability of failure is defined, for any subdomain $\mathcal{H} \subset \mathcal{D}$ by [11]:

$$
P_{f}(\mathcal{H}, t)=\mathbb{P}(\exists \boldsymbol{x} \in \mathcal{H} \quad, \quad g(\boldsymbol{Z}(\boldsymbol{x}), t) \leq 0)=\mathbb{P}\left(\bigcup_{\boldsymbol{x} \in \mathcal{H}} g(\boldsymbol{Z}(\boldsymbol{x}), t) \leq 0\right)
$$

This quantity is the "spatial" counterpart of the so-called cumulative probability of failure in timevariant reliability problems [15]. 
When the damage measure is related to serviceability of the structure (e.g. apparition of cracks or rebars loss of diameter) and not directly related to the collapse of the structure, none of the above quantities are sufficient to characterize the global state of ageing of the structure. Indeed, Eq.(6) is by definition a local quantity (at point $\boldsymbol{x}$ ). Eq.(7) refers to the probability that there is at least one point in subdomain $\mathcal{H}$ where the local damage criterion is attained. This probability is likely to be close to one, without meaning that the structure is close to structural failure.

In contrast, the extent of damage is of major interest, especially for the comparison of maintenance policies, see e.g. $[5,8]$

\section{Extent of damage}

\subsection{Definition}

The extent of damage is defined at each time instant $t$ as the measure of the subdomain of $\mathcal{D}$ in which the local failure criterion is attained:

$$
\mathcal{E}(\mathcal{D}, t)=\int_{\mathcal{D}} \mathbf{1}_{\{g(\boldsymbol{Z}(\boldsymbol{x}), t) \leq 0\}}(\boldsymbol{x}) d \boldsymbol{x}
$$

Note that $\mathcal{E}(\mathcal{D}, t)$ is a scalar random variable since the integral over $\boldsymbol{x}$ is defined for each realization of the input random field, say $\boldsymbol{z}(\boldsymbol{x})$. It is positive-valued and is by definition bounded by the volume of the structure in $\mathbb{R}^{d}$ denoted by $|\mathcal{D}|$. Again, due to the monotony of degradation phenomena, each realization of $\mathcal{E}(\mathcal{D}, t)$, say $\mathfrak{e}(\mathcal{D}, t)$ is a continuously increasing function of time.

\subsection{Mean and variance}

By taking the expectation of Eq.(8) (i.e. with respect to $Z$ ), one gets the following expression for the mean value of the extent of damage:

$$
\overline{\mathcal{E}(\mathcal{D}, t)} \equiv \mathrm{E}[\mathcal{E}(\mathcal{D}, t)]=\int_{\mathcal{D}} \mathrm{E}\left[\mathbf{1}_{\{g(\boldsymbol{Z}(\boldsymbol{x}), t) \leq 0\}}(\boldsymbol{x})\right] d \boldsymbol{x}
$$

By comparing the integrand of the above equation with Eq.(6), one gets:

$$
\overline{\mathcal{E}(\mathcal{D}, t)}=\int_{\mathcal{D}} P_{f}(\boldsymbol{x}, t) d \boldsymbol{x}
$$

In case of homogeneous input random field, this integrand is independent of $\boldsymbol{x}$, as explained above. Thus:

$$
\overline{\mathcal{E}(\mathcal{D}, t)}=P_{f}\left(\boldsymbol{x}_{0}, t\right) \cdot|\mathcal{D}| \quad \text { (Homogeneous case) }
$$

where the point-in-space probability of failure is computed at any point $\boldsymbol{x}_{0} \in \mathcal{D}$.

The above equation has the following interpretation: the proportion of the structure where the damage criterion is attained (i.e. $\overline{\mathcal{E}(\mathcal{D}, t)} /|\mathcal{D}|)$ is, in the mean, equal to the point-in-space probability of failure. This remark has two important consequences:

- it is not necessary to introduce the complex formalism of random fields when one is interested only in the mean value of $\overline{\mathcal{E}(\mathcal{D}, t)}$. Only the description of the input random variables gathered in vector $\boldsymbol{Z}$ is required. 
- the mean proportion of the structure that is damaged is independent of the correlation structure of the input random field $\boldsymbol{Z}(\boldsymbol{x})$, if the spatial variability is modelled. This is a valuable result, since the determination of the correlation structure is difficult and hardly done in practice, due to the lack of data (the auto-correlation functions and their parameters being often chosen from "expert judgment", see e.g. $[5,8])$.

In order to better capture the probabilistic content of $\mathcal{E}(\mathcal{D}, t)$, it is useful to study the variance of the extent of damage. By definition, this quantity reads:

$$
\operatorname{Var}[\mathcal{E}(\mathcal{D}, t)]=\mathrm{E}\left[\mathcal{E}^{2}(\mathcal{D}, t)\right]-\overline{\mathcal{E}(\mathcal{D}, t)}^{2}
$$

From the definition in Eq.(8) one can write:

$$
\begin{aligned}
\mathcal{E}^{2}(\mathcal{D}, t) & =\left(\int_{\mathcal{D}} \mathbf{1}_{\{g(\boldsymbol{Z}(\boldsymbol{x}), t) \leq 0\}}(\boldsymbol{x}) d \boldsymbol{x}\right) \cdot\left(\int_{\mathcal{D}} \mathbf{1}_{\{g(\boldsymbol{Z}(\boldsymbol{x}), t) \leq 0\}}(\boldsymbol{x}) d \boldsymbol{x}\right) \\
& =\int_{\mathcal{D}} \int_{\mathcal{D}} \mathbf{1}_{\left\{g\left(\boldsymbol{Z}\left(\boldsymbol{x}_{1}\right), t\right) \leq 0\right\}}\left(\boldsymbol{x}_{1}\right) \cdot \mathbf{1}_{\left\{g\left(\boldsymbol{Z}\left(\boldsymbol{x}_{2}\right), t\right) \leq 0\right\}}\left(\boldsymbol{x}_{2}\right) d \boldsymbol{x}_{1} d \boldsymbol{x}_{2}
\end{aligned}
$$

The integrand is equal to one if and only if the limit state function takes negative values at both locations $\boldsymbol{x}_{1}$ and $\boldsymbol{x}_{2}$. Thus (13) may be rewritten as:

$$
\mathcal{E}^{2}(\mathcal{D}, t)=\int_{\mathcal{D}} \int_{\mathcal{D}} \mathbf{1}_{\left\{g\left(\boldsymbol{Z}\left(\boldsymbol{x}_{1}\right), t\right) \leq 0 \cap g\left(\boldsymbol{Z}\left(\boldsymbol{x}_{2}\right), t\right) \leq 0\right\}}\left(\boldsymbol{x}_{1}, \boldsymbol{x}_{2}\right) d \boldsymbol{x}_{1} d \boldsymbol{x}_{2}
$$

Hence:

$$
\mathrm{E}\left[\mathcal{E}^{2}(\mathcal{D}, t)\right]=\int_{\mathcal{D}} \int_{\mathcal{D}} \mathbb{P}\left(g\left(\boldsymbol{Z}\left(\boldsymbol{x}_{1}\right), t\right) \leq 0 \cap g\left(\boldsymbol{Z}\left(\boldsymbol{x}_{2}\right), t\right) \leq 0\right) d \boldsymbol{x}_{1} d \boldsymbol{x}_{2}
$$

This equation is similar to the results obtained by Koo and Der Kiureghian [16] for the excursion time in the context of first-passage problem in time-variant reliability analysis.

Here again, the assumption of homogeneity allows to simplify the result. Indeed, the integrand in Eq.(15) depends only on $\left|\boldsymbol{x}_{1}-\boldsymbol{x}_{2}\right|$ in this case, meaning that it is an even function of $\left(x_{1}^{j}-x_{2}^{j}\right), j=$ $1, \ldots, d$. One can prove that the above double integral may be reduced to a single integral (see details in Appendix A) and further simplified. For the sake of clarity, results are reported here separately for $d=1$ and $d=2$.

- For a beam of length $L(d=1,|\mathcal{D}|=L)$, the variance of the extent of damage is:

$$
\operatorname{Var}[\mathcal{E}(\mathcal{D}, t)]=L^{2} \int_{0}^{1} \mathbb{P}(g(\boldsymbol{Z}(0), t) \leq 0 \cap g(\boldsymbol{Z}(L u), t) \leq 0)(2-2 u) d u-\overline{\mathcal{E}(\mathcal{D}, t)}^{2}
$$

- For a rectangular plate of dimensions $\left(L_{1}, L_{2}\right)$, the variance of the extent of damage is:

$$
\begin{gathered}
\operatorname{Var}[\mathcal{E}(\mathcal{D}, t)]=L_{1}^{2} L_{2}^{2} \int_{0}^{1} \int_{0}^{1} \mathbb{P}\left(g(\boldsymbol{Z}(0,0), t) \leq 0 \cap g\left(\boldsymbol{Z}\left(L_{1} u, L_{2} v\right), t\right) \leq 0\right) \ldots \\
\ldots(2-2 u)(2-2 v) d u d v \\
\quad-\overline{\mathcal{E}(\mathcal{D}, t)}^{2}
\end{gathered}
$$

The integrals in Eqs.(16),(17) are rather easy to evaluate since both the integration domain and the integrands are bounded. A typical Gaussian quadrature rule [17] can be applied, as shown in the next section. 


\subsection{Conclusion}

In this section, the mean and standard deviation of the extent of damage have been derived in a closed form. The obtained formulæ do not require that the random fields describing the spatial variability of the problem are discretized. It has been shown that the mean value of the extent of damage does not depend on the correlation structure of the input random fields. In case of homogeneous input, it may be computed from a single point-in-space analysis (Eq.(11)).

In case of $1 \mathrm{D}$ or $2 \mathrm{D}$-rectangular structures, the computation of the variance further reduces to a single integral over $\mathcal{D}$ instead of a double integral. These cases are of great practical importance, since the majority of civil engineering structures may be decomposed into beams and plates. Eq.(17) is also applicable to shell structures (e.g. cooling towers) as soon as the geometry of these structures is parametrized by a rectangular domain, e.g. $\left\{(\theta, z), \theta \in[0,2 \pi], z \in\left[0, z_{\max }\right]\right\}$.

\section{Numerical implementation}

As explained above, the mean and standard deviation of the extent of damage may be computed from Eqs.(11),(16),(17) without discretizing the input random fields. The practical implementation of these equations is described in the next subsection.

In order to assess the accuracy of the proposed approach, the results have to be compared with the Monte Carlo simulation of the extent of damage. For this purpose, the input random fields shall be discretized and simulated. This is described in the second next subsection.

\subsection{Analytical approach}

We consider here the computation of mean and standard deviation of the extent of damage (also called damage length in the one-dimensional case) by the analytical equations Eqs.(11),(16). The extension to (17) is straightforward.

As shown in the previous section, Eq.(11) reduces to solving a time-invariant point-in-space reliability problem. The first order reliability method (FORM) may be used for this purpose.

In order to evaluate Eq.(16), note that the probability under the integral is nothing but the parallel system failure probability associated to the events $\{g(\boldsymbol{Z}(0), t) \leq 0\}$ and $\{g(\boldsymbol{Z}(L u), t) \leq 0\}$. This probability may be computed using the FORM method applied to parallel systems:

$$
\mathbb{P}(g(\boldsymbol{Z}(0), t) \leq 0 \cap g(\boldsymbol{Z}(L u), t)) \approx \Phi_{2}\left(-\beta(0, t),-\beta(L v, t), \rho_{g}(L u, t)\right)
$$

In this equation, the following notation is used:

- $\Phi_{2}(x, y, \rho)$ is the binormal CDF;

- $\beta(0, t)$ (resp. $\beta(L u, t)$ ) is the point-in-space reliability index at abscissa $x=0$ (resp. abscissa $x=L u$ ). Note that both values are equal when homogeneous fields are considered.

- $\rho_{g}(L u, t)=\boldsymbol{\alpha}(0, t) \cdot \boldsymbol{\alpha}(L u, t)$ is the dot product of the $\boldsymbol{\alpha}$-vectors obtained by FORM. Remember that the $\alpha$-vector is the unit vector in the direction of the design point in the standard normal space. 
Eq.(18) is practically evaluated as follows:

- a point-in-space FORM analysis is carried out at $x=0$, replacing the random fields by a set of random variables having the same PDF, say $\boldsymbol{Z}^{(1)}$.

- another point-in-space FORM analysis is carried out at $x=L u$, replacing the random fields by another set of random variables having the same PDF, say $\boldsymbol{Z}^{(2)}$. If some components $Z_{j}$ are actually random variables (in other words, fully correlated fields), the same variables are used in both analysis $\left(Z_{j}^{(1)}=Z_{j}^{(2)}\right)$. Otherwise, the components of $\boldsymbol{Z}^{(2)}$ corresponding to "true" random fields are correlated to the corresponding components of $\boldsymbol{Z}^{(1)}$ using the random field autocorrelation matrix.

- the reliability indices and $\boldsymbol{\alpha}$-vectors obtained by both analysis are reported in Eq.(18).

As a conclusion, the evaluation of Eq.(18) requires two FORM analysis. Finally, the integral in Eq.(16) may be evaluated using a Gaussian quadrature formula. The principle of Gaussian integration is to approximate the integral by a weighted summation:

$$
\int_{0}^{1} h(u) d u=\int_{-1}^{1} h\left(\frac{1+s}{2}\right) \frac{d s}{2} \approx \sum_{i=1}^{K} \frac{\omega_{i}}{2} h\left(\frac{1+s_{i}}{2}\right)
$$

where $\left\{\left(\omega_{i}, s_{i}\right), i=1, \ldots, K\right\}$ are the integration weights and points [17]. Again, the extension of the latter equation to two-dimensional problems $(d=2$, see Eq.(17)) is straightforward.

\subsection{EOLE method for random field discretization}

In order to compute the mean and standard deviation of the extent of damage by Monte Carlo simulation, the simulation of spatial realizations of the damage criterion is required. This can be done after a proper discretization of the input random fields. The expansion optimal linear estimation method (EOLE) [18] is used for this purpose in case of Gaussian random fields (it is assumed that the components of the vector input random field are independent). It may then be also applied to discretize fields obtained by translation, e.g. lognormal fields. The method is based on the pointwise regression of the original random field with respect to selected values of the field, and a compaction of the data by spectral analysis. The method is now recalled.

Let us consider a scalar Gaussian random field $H(\boldsymbol{x})$ defined by its mean $\mu(\boldsymbol{x})$, its standard deviation $\sigma(\boldsymbol{x})$ and its autocorrelation coefficient function $\rho\left(\boldsymbol{x}_{1}, \boldsymbol{x}_{2}\right)$. Let us consider a grid of points $\left\{\boldsymbol{x}_{1}, \ldots \boldsymbol{x}_{N}\right\}$ in $\mathcal{D}$ and denote by $\boldsymbol{\chi}$ the random vector $\left\{H\left(\boldsymbol{x}_{1}\right), \ldots H\left(\boldsymbol{x}_{N}\right)\right\}$. By construction, $\boldsymbol{\chi}$ is a Gaussian vector whose mean value $\boldsymbol{\mu}_{\chi}$ and covariance matrix $\boldsymbol{\Sigma}_{\chi \chi}$ read:

$$
\begin{aligned}
\boldsymbol{\mu}_{\chi}^{i} & =\mu\left(\boldsymbol{x}_{i}\right) \\
\left(\boldsymbol{\Sigma}_{\chi \chi}\right)_{i, j} & =\operatorname{Cov}\left[H\left(\boldsymbol{x}_{i}\right), H\left(\boldsymbol{x}_{j}\right)\right]=\sigma\left(\boldsymbol{x}_{i}\right) \sigma\left(\boldsymbol{x}_{j}\right) \rho\left(\boldsymbol{x}_{i}, \boldsymbol{x}_{j}\right)
\end{aligned}
$$

The optimal linear estimation (OLE) of random variable $H(\boldsymbol{x})$ onto the random vector $\chi$ reads:

$$
H(\boldsymbol{x}) \approx \hat{H}(\boldsymbol{x})=\mu(\boldsymbol{x})+\boldsymbol{\Sigma}_{H \chi}^{\prime}(\boldsymbol{x}) \cdot \boldsymbol{\Sigma}_{\chi \chi}^{-1} \cdot\left(\chi-\boldsymbol{\mu}_{\chi}\right)
$$

where (.) $)^{\prime}$ denotes the transposed matrix and $\boldsymbol{\Sigma}_{H \chi}(\boldsymbol{x})$ is a vector whose component are given by:

$$
\boldsymbol{\Sigma}_{H \chi}^{j}(\boldsymbol{x})=\operatorname{Cov}\left[H(\boldsymbol{x}), \chi_{j}\right]=\operatorname{Cov}\left[H(\boldsymbol{x}), H\left(\boldsymbol{x}_{j}\right)\right]=\sigma(\boldsymbol{x}) \sigma\left(\boldsymbol{x}_{j}\right) \rho\left(\boldsymbol{x}, \boldsymbol{x}_{j}\right)
$$


Let us now consider the spectral decomposition of the covariance matrix $\Sigma_{\chi \chi}$ :

$$
\boldsymbol{\Sigma}_{\chi \chi} \phi_{i}=\lambda_{i} \phi_{i} \quad i=1, \ldots, N
$$

This allows to linearly transform the original vector $\chi$ :

$$
\chi=\boldsymbol{\mu}_{\chi}+\sum_{i=1}^{N} \sqrt{\lambda_{i}} \xi_{i} \phi_{i}
$$

where $\left\{\xi_{i}, i=1, \ldots N\right\}$ are independent standard normal variables. Substituting for (25) in (22) and using (24) yields the OLE representation of the field :

$$
\hat{H}(\boldsymbol{x})=\mu(\boldsymbol{x})+\sum_{i=1}^{N} \frac{\xi_{i}}{\sqrt{\lambda_{i}}} \phi_{\boldsymbol{i}}{ }^{\prime} \cdot \boldsymbol{\Sigma}_{H(\boldsymbol{x}) \chi}(\boldsymbol{x}) \quad \text { (OLE expansion) }
$$

The series can be further truncated after $r \leq N$ terms, the eigenvalues $\lambda_{i}$ being sorted first in descending order. This yields the EOLE expansion:

$$
\hat{H}(\boldsymbol{x})=\mu(\boldsymbol{x})+\sum_{i=1}^{r} \frac{\xi_{i}}{\sqrt{\lambda_{i}}} \boldsymbol{\phi}_{\boldsymbol{i}}{ }^{\prime} \cdot \boldsymbol{\Sigma}_{H(\boldsymbol{x}) \chi}(\boldsymbol{x}) \quad \text { (EOLE expansion) }
$$

The variance of the error for the EOLE discretization reads:

$$
\operatorname{Var}[H(\boldsymbol{x})-\hat{H}(\boldsymbol{x})]=\sigma^{2}(\boldsymbol{x})-\sum_{i=1}^{r} \frac{1}{\lambda_{i}}\left(\boldsymbol{\phi}_{i}^{\prime} \cdot \boldsymbol{\Sigma}_{H(\boldsymbol{x}) \chi}(\boldsymbol{x})\right)^{2}
$$

The above equation allows to check that the grid density (i.e. number of points $N$ ) and the number of terms $r$ are large enough to attain a prescribed accuracy in the discretization. Details on how choosing these parameters are given in [19].

Example Let us consider a homogeneous univariate $(d=1)$ standard normal random field (with zero mean and unit standard deviation) having one of the following autocorrelation coefficient function:

- Type A:

$$
\rho_{A}\left(x_{1}, x_{2}\right)=\exp \left(-\left|\frac{x_{1}-x_{2}}{\ell_{A}}\right|\right)
$$

- Type B:

$$
\rho_{B}\left(x_{1}, x_{2}\right)=\exp \left(-\left(\frac{x_{1}-x_{2}}{\ell_{B}}\right)^{2}\right)
$$

- Type C:

$$
\rho_{C}\left(x_{1}, x_{2}\right)=\frac{\sin \left(\left(x_{1}-x_{2}\right) / \ell_{C}\right)}{\left(x_{1}-x_{2}\right) / \ell_{C}}
$$

In order to study the influence of the shape of the autocorrelation function, it is desirable to find an equivalence between the various parameters. The usual measure is the scale of fluctuation proposed by Vanmarcke [20]:

$$
\theta=2 \int_{0}^{\infty} \rho(0, x) d x
$$




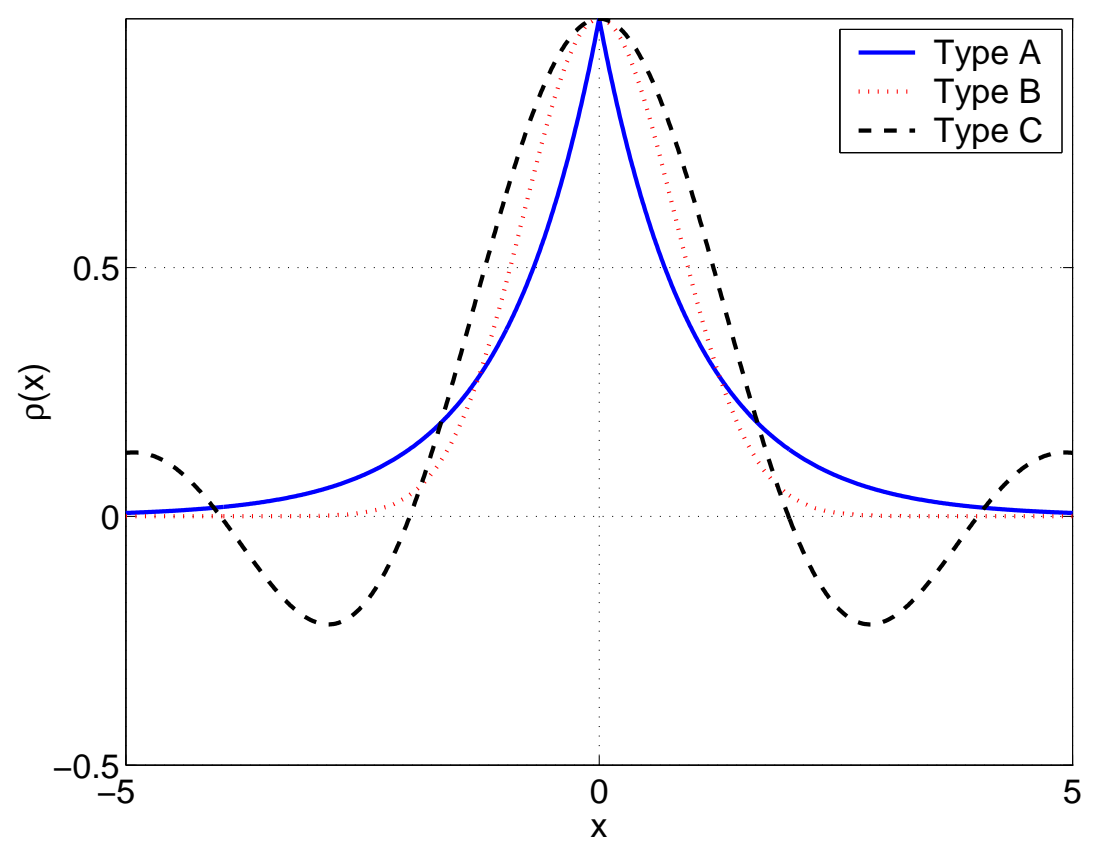

Figure 1: Autocorrelation coefficient functions (scale of fluctuation $\theta=2$ )

For the three types of autocorrelation coefficient functions given above, the results are:

$$
\begin{aligned}
& \theta_{A}=2 \ell_{A} \\
& \theta_{B}=\sqrt{\pi} \ell_{B} \\
& \theta_{C}=\pi \ell_{C}
\end{aligned}
$$

The three autocorrelation coefficient functions are plotted in Figure 1 for $\theta=2$. The random field is now discretized over the interval $x \in[0,10]$ using $N=81$ points (regular grid with stepsize equal to $10 / 80=0.125)$. A number $r=16$ terms is retained in the EOLE expansion. Figure 2 presents the evolution of the variance error (Eq.(28)) as a function of $x \in[0,10]$ for the three functions. Obviously the discretization scheme is not accurate enough for Type A correlation (maximal error of 16.7\%), accurate enough for Type B (maximal error of $0.1 \%$ ) and almost exact for Type $\mathrm{C}$ (maximal error of $\left.10^{-13} \%\right)$.

\subsection{Monte Carlo simulation of the extent of damage}

Once an input random field, say $Z_{j}(\boldsymbol{x})$ has been discretized using EOLE, a realization of the field $\hat{Z}_{j}^{o}(\boldsymbol{x})$ (i.e. a usual function of $\boldsymbol{x} \in \mathcal{D}$ ) is obtained by simulating a set of $r$ standard normal random variables, say $\left\{\xi_{1}^{o}, \ldots \xi_{r}^{o}\right\}$, and substituting for them in Eq.(27):

$$
\hat{Z}_{j}^{o}(\boldsymbol{x})=\mu(\boldsymbol{x})+\sum_{i=1}^{r} \frac{\xi_{i}^{o}}{\sqrt{\lambda_{i}}} \boldsymbol{\phi}_{\boldsymbol{i}}{ }^{\prime} \cdot \boldsymbol{\Sigma}_{Z_{j}(\boldsymbol{x}) \chi}(\boldsymbol{x})
$$

The above spatial realizations can then be used to get spatial realizations of the limit state function $g\left(\boldsymbol{Z}^{o}(\boldsymbol{x}), t\right)$ at time instant $t$. Each realization shall be post-processed in order to compute the realization of the extent of damage $\mathcal{E}_{o}(\mathcal{D}, t)$. 


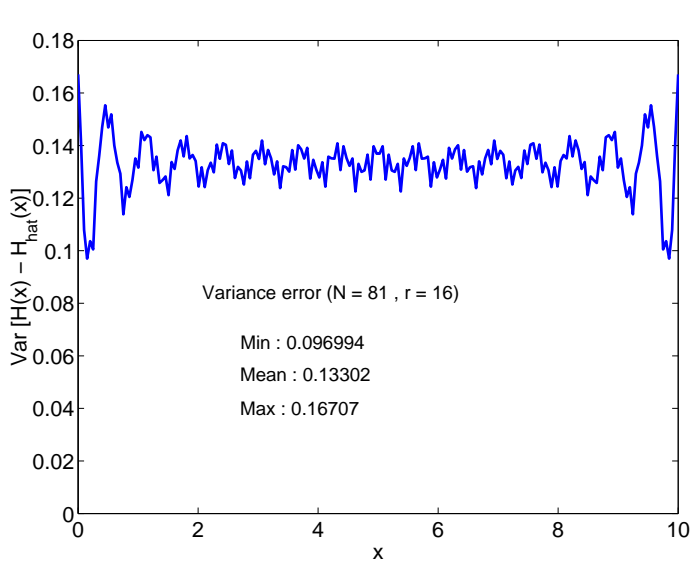

(a) Type A

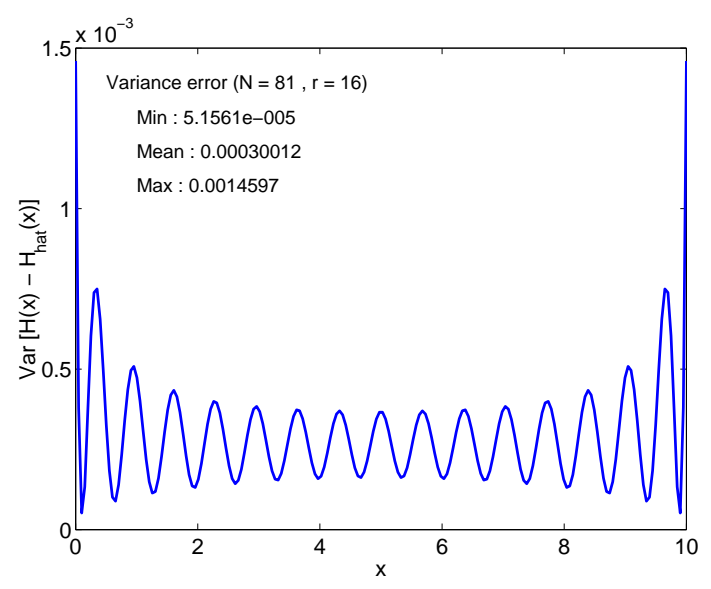

(b) Type $B$

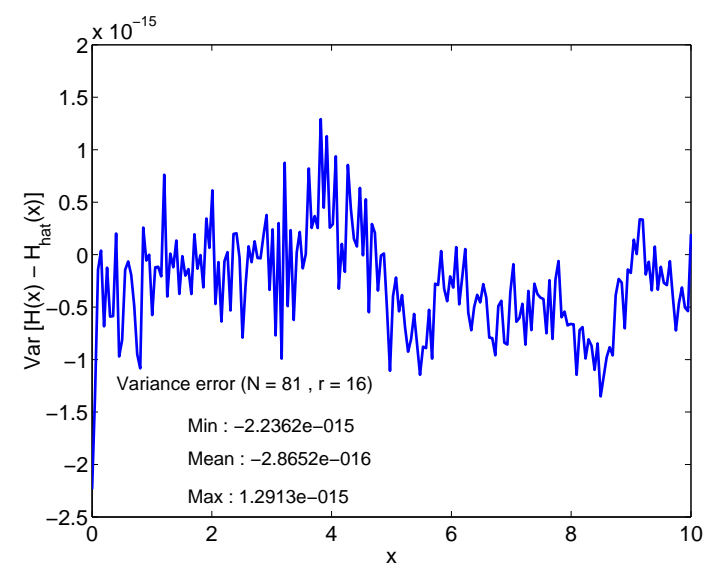

(c) Type $C$

Figure 2: Variance of the error of discretization for various autocorrelation coefficient functions $(x \in[0,10], \theta=2, N=81, r=16)$

In the recent literature on space-variant probabilistic damage models, see e.g. $[5,6,8]$, the so-called midpoint approach (MP), originally proposed in [21], has been used to discretize the random fields and post-process the extent of damage. It is important to recall that this crude approach is by far less accurate than the EOLE decomposition, even when using a large number $N_{M P}$ of elements in the midpoint approach (the comparaison of accuracy can be found in $[18,19]$ ).

Moreover, the midpoint approach also provides a rough estimation of the extent of damage for each trajectory. Indeed, if $N_{M P}$ elements have been used for the discretization, the extent of damage for a given realization of the input fields is estimated by $\frac{N_{D}}{N_{M P}}|\mathcal{D}|$, where $N_{D}$ is the number of elements where the damage criterion $g\left(\boldsymbol{Z}^{o}(\boldsymbol{x}), t\right)$ is attained. As $N_{M P}$ is limited in practice, since it is also equal to the number of random variables required for discretizing each field, the above estimate may be crude.

In contrast, the EOLE method, which provides smooth realizations of the field may be post-processed efficiently. In the following, the one-dimensional $(d=1, \mathcal{D}=[0, L])$ case is presented, the extension to larger dimensions being straightforward, although quite technical. Each realization of the damage 
criterion is stored in an array of size $Q+1$ corresponding to the evaluation of $g\left(\boldsymbol{Z}^{o}(\boldsymbol{x}), t\right)$ at selected points:

$$
\left\{g_{j}=g\left(\boldsymbol{Z}^{o}(j L / Q), t\right), \quad j=0, \ldots Q\right\}
$$

The damage length is then obtained by screening this array according to the algorithm summarized in Figure 3. If, for a given $j \in[0, Q-1], g_{j} \leq 0$ and $g_{j+1} \leq 0$ it is supposed that the failure criterion is negative for any $x \in[j L / Q,(j+1) L / Q]$ and the damage length is increased by $L / Q$. If $g$ changes its sign between $j L / Q$ and $(j+1) L / Q$, a linear interpolation allows to compute the portion of the latter interval where $g$ is negative. Of course, $Q$ has to be selected in such a way that the trajectories do not change sign twice within an interval of the form $[j L / Q,(j+1) L / Q]$, i.e. consistently with the scales of fluctuation of the input fields.

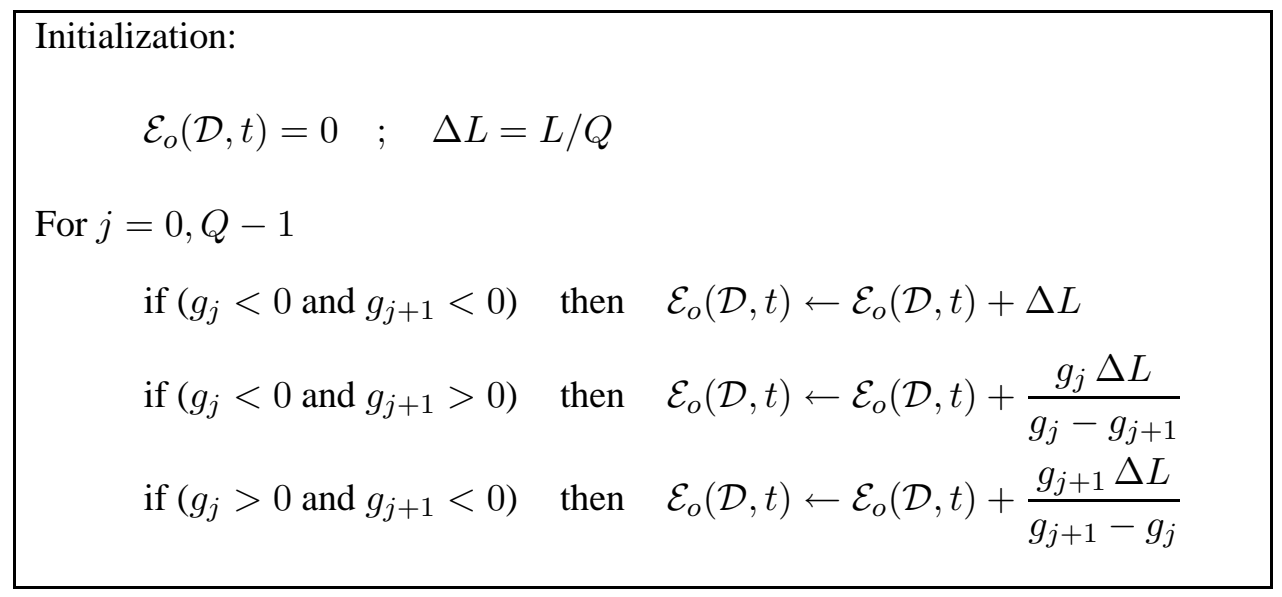

Figure 3: Algorithm for computing the extent of damage by Monte Carlo simulation

\section{Application example: corrosion induced by concrete carbonation}

\subsection{Point-in-space model of carbonation}

Concrete carbonation is a complex physico-chemical process that includes the diffusion of $\mathrm{CO}_{2}$ into the gas phase of the concrete pores and its reaction with the calcium hydroxyl $\mathrm{Ca}(\mathrm{OH})_{2}$. The latter can be simplified into:

$$
\mathrm{Ca}(\mathrm{OH})_{2}+\mathrm{CO}_{2} \longrightarrow \mathrm{CaCO}_{3}+\mathrm{H}_{2} \mathrm{O}
$$

As the high $\mathrm{pH}$ of uncarbonated concrete is mainly due to the presence of $\mathrm{Ca}(\mathrm{OH})_{2}$, it is clear that the consumption of this species will lead to a $\mathrm{pH}$ drop, which can attain a value of 9 when the reaction is completed. In this environment, the oxide layer that protected the reinforcement bars is attacked and corrosion starts. The corrosion products tend to expand into the pores of concrete, developping tensile stresses which eventually lead to cracking [22, 23, 24].

In practice, $\mathrm{CO}_{2}$ penetrates into the concrete mass by diffusion from the surface layer. Thus a carbonation front appears that moves into the structure. A model for computing the carbonation depth $x_{c}$ is proposed by the CEB Task Groups $5.1 \& 5.2$ [25]. The simplified version retained in the present paper reads:

$$
x_{c}(t)=\sqrt{\frac{2 C_{0} D_{C O_{2}}}{a} t}
$$


where $D_{\mathrm{CO}_{2}}$ is the coefficient of diffusion of carbon dioxide in dry concrete, $C_{0}$ is the carbon dioxide concentration in the surrounding air and $a$ is the binding capacity, i.e. the amount of carbon dioxide necessary for complete carbonation of a concrete volume. It is supposed that corrosion immediately starts when carbonation has attained the rebars. Denoting by $e$ the concrete cover, the time necessary for corrosion to start, called initiation time, reads:

$$
T_{\text {init }}=\frac{a e^{2}}{2 C_{0} D_{C O_{2}}}
$$

If generalized corrosion is considered, the loss of metal due to corrosion is approximately uniform over the whole surface. In this case, Faraday's law indicates that a unit corrosion current density (or corrosion rate) corresponds to a uniform corrosion penetration of $\kappa=11,6 \mu \mathrm{m} / \mathrm{year}$. If a constant annual corrosion rate is supposed, the expression of the rebars diameter as a function of time eventually reads:

$$
\phi(t)=\left\{\begin{array}{l}
\phi_{0} \quad \text { if } t \leq T_{\text {init }} \\
\max \left[\phi_{0}-2 i_{\text {corr }} \kappa\left(t-T_{\text {init }}\right), 0\right] \quad \text { if } t>T_{\text {init }}
\end{array}\right.
$$

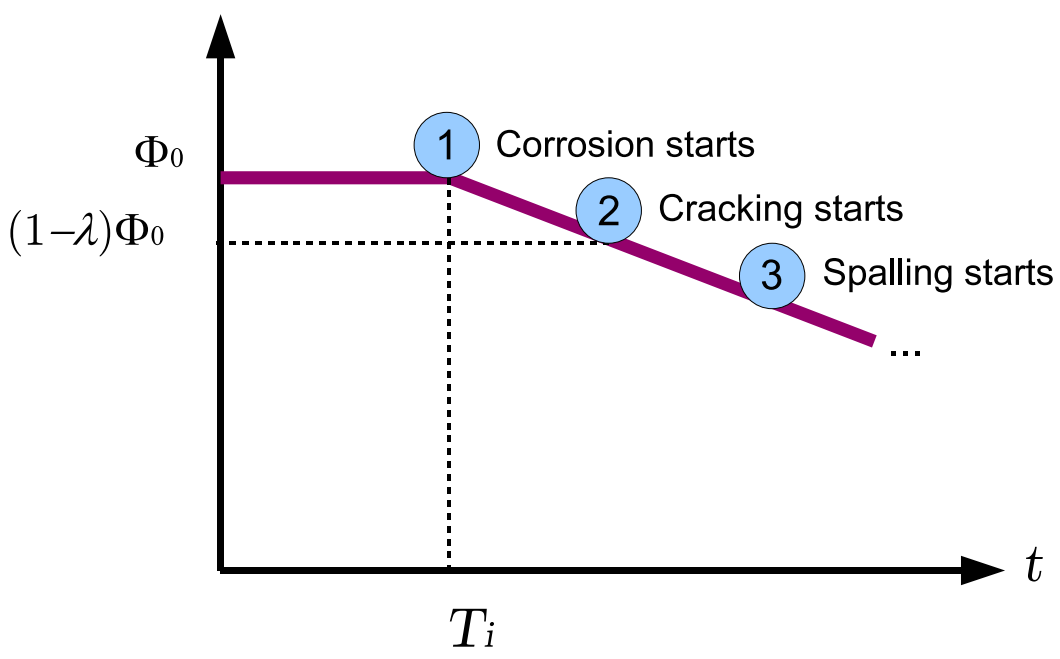

Figure 4: Evolution in time of the rebars diameter and associated state of damage

Figure 4 sketches the evolution in time of the rebars diameter together with the associated damage on the structure. From experimental evidence [26, 27], it is possible to associate a value $\lambda$ (representing the relative loss of rebar's diameter) to a given state of damage (i.e. crack initiation, severe cracking, spalling, etc.). For instance, a value of $\lambda=0.5-1 \%$ is consistent with the apparition of cracks.

In a probabilistic context, the random parameters are those appearing in Eqs.(38),(39), namely the coefficient of diffusion of carbon dioxide $D_{\mathrm{CO}_{2}}$, the surface carbon dioxide concentration $C_{0}$, the binding capacity $a$, the concrete cover $e$, the rebars initial diameter $\phi_{0}$ and the corrosion current density $i_{\text {corr }}$ :

$$
\boldsymbol{Z}=\left\{D_{\mathrm{CO}_{2}}, C_{0}, a, e, \phi_{0}, i_{\text {corr }}\right\}
$$

The damage criterion is defined at a given time instant by the fact that the residual rebars diameter (Eq.(39)) becomes smaller than a prescribed fraction $(1-\lambda)$ of its initial value:

$$
g(\boldsymbol{Z}, t)=\phi(t)-(1-\lambda) \phi_{0}
$$


Remarking that Eq.(39) rewrites:

$$
\phi(t)=\min \left(\phi_{0}, \phi_{0}-2 i_{\text {corr }} \kappa\left(t-T_{\text {init }}\right)\right)
$$

for reasonable values of $t$ that do not lead to "negative" values of $\phi(t)$, Eq.(41) becomes:

$$
g(\boldsymbol{Z}, t)=\min \left(\lambda \phi_{0}, \lambda \phi_{0}-2 i_{\text {corr }} \kappa\left(t-T_{\text {init }}\right)\right)
$$

Thus the probability of failure may be interpreted as that of a series system:

$$
P_{f}(t)=\mathbb{P}\left(\left\{\lambda \phi_{0} \leq 0\right\} \cup\left\{\lambda \phi_{0}-2 i_{\text {corr }} \kappa\left(t-T_{\text {init }}\right) \leq 0\right\}\right)
$$

As the rebars diameter is positive in nature, the above system event reduces to its second component. Thus:

$$
P_{f}(t)=\mathbb{P}\left(\lambda \phi_{0}-2 i_{\text {corr }} \kappa\left(t-T_{\text {init }}\right) \leq 0\right)
$$

Following these remarks and using (38)), the limit state function (41) eventually reads:

$$
g(\boldsymbol{Z}, t)=\lambda \phi_{0}-2 i_{\text {corr }} \kappa\left(t-\frac{a e^{2}}{2 C_{0} D_{C O_{2}}}\right)
$$

\subsection{Probabilistic problem statement}

In order to illustrate the concept of extent of damage described above, we consider a concrete beam of length $L=10 \mathrm{~m}$. This beam is reinforced by a single longitudinal reinforcing bar whose initial diameter is modelled by a lognormal random variable $\phi_{0}$. The concrete cover $e(x)$ is a univariate homogeneous lognormal random field, obtained by exponentiation of a Gaussian random field, whose properties are given below. This allows to model the imperfections in placing the rebar into the falsework.

The parameters $\left\{C_{0}, a\right\}$ are modelled by lognormal random variables. The coefficient of diffusion $D_{\mathrm{CO}_{2}}$ is modelled as a homogeneous lognormal random field. The corrosion current density $i_{\text {corr }}(x)$ is supposed to be inversely proportional to the concrete cover (see [6]):

$$
i_{\text {corr }}(x)=i_{\text {corr }}^{0} \frac{e_{0}}{e(x)}
$$

In this equation, $e_{0}=50 \mathrm{~mm}$ is the mean concrete cover and $i_{c o r r}^{0}$ is a lognormal random variable. The above expression has the following interpretation: variable $i_{\text {corr }}^{0}$ models the overall uncertainty on the corrosion rate, whereas its spatial variability is perfectly correlated to that of the concrete cover. The mean value of the corrosion rate is $2.08 \mu \mathrm{A} / \mathrm{cm}^{2}$. The parameters describing these six input quantities ( 2 random fields and 4 random variables) are gathered in Table 1.

\subsection{Mean and variance of the extent of damage}

The mean value of the damage length is computed by three approaches:

- Eq.(11), where the point-in-space probability of failure, obtained by freezing the spatial coordinate $x$ (i.e. replacing the random fields $e(x)$ and $D_{\mathrm{CO}_{2}}(x)$ by lognormal random variables) is computed by FORM analysis; 
Table 1: Probabilistic input data

\begin{tabular}{lcccc}
\hline Parameter & Type of PDF & Mean value & Coef. Var. & A.c.f $^{\dagger}$ \\
\hline Rebars diameter $\phi_{0}$ & lognormal & $10 \mathrm{~mm}$ & $10 \%$ & - \\
Diffusion coefficient $D_{C O_{2}}$ & lognormal & $5.10^{-8} \mathrm{~m}^{2} / \mathrm{s}$ & $30 \%$ & $\rho_{D}\left(x_{1}, x_{2}\right)$ \\
Surface concentration $C_{0}$ & lognormal & $6.210^{-4} \mathrm{~kg} / \mathrm{m}^{3}$ & $30 \%$ & - \\
Binding capacity $a$ & lognormal & $80 \mathrm{~kg} / \mathrm{m}^{3}$ & $30 \%$ & - \\
Nominal corrosion rate $i_{\text {corr }}^{0}(x)$ & lognormal & $1 \mu A / \mathrm{cm}^{2}$ & $25 \%$ & - \\
Concrete cover $e(s)$ & lognormal & $50 \mathrm{~mm}$ & $20 \%$ & $\rho_{e}\left(x_{1}, x_{2}\right)$ \\
\hline
\end{tabular}

${ }^{\dagger}$ Autocorrelation coefficient function of the underlying Gaussian field $\rho_{D}\left(x_{1}, x_{2}\right)=e^{-\pi\left(x_{1}-x_{2}\right)^{2} / \theta_{D}^{2}}, \theta_{D}=2 \mathrm{~m} ; \rho_{e}\left(x_{1}, x_{2}\right)=e^{-\pi\left(x_{1}-x_{2}\right)^{2} / \theta_{e}^{2}}, \theta_{e}=2 \mathrm{~m}$

- Eq.(11), where the point-in-space probability of failure is obtained by Monte Carlo simulation $(100,000$ samples were used);

- The field discretization approach described in Section 4.3 and Figure 3. The EOLE discretization of both input fields was carried out using a regular grid consisting in $N=81$ points over $[0,10 \mathrm{~m}]$. A number $r=16$ points was retained in the spectral decomposition (Eq.(27)) . This allows to get a maximal relative discretization error on the field variance of $0.1 \%$ (as shown in Figure 2(b) above). A total number of 10,000 spatial realizations of the limit state function was used.

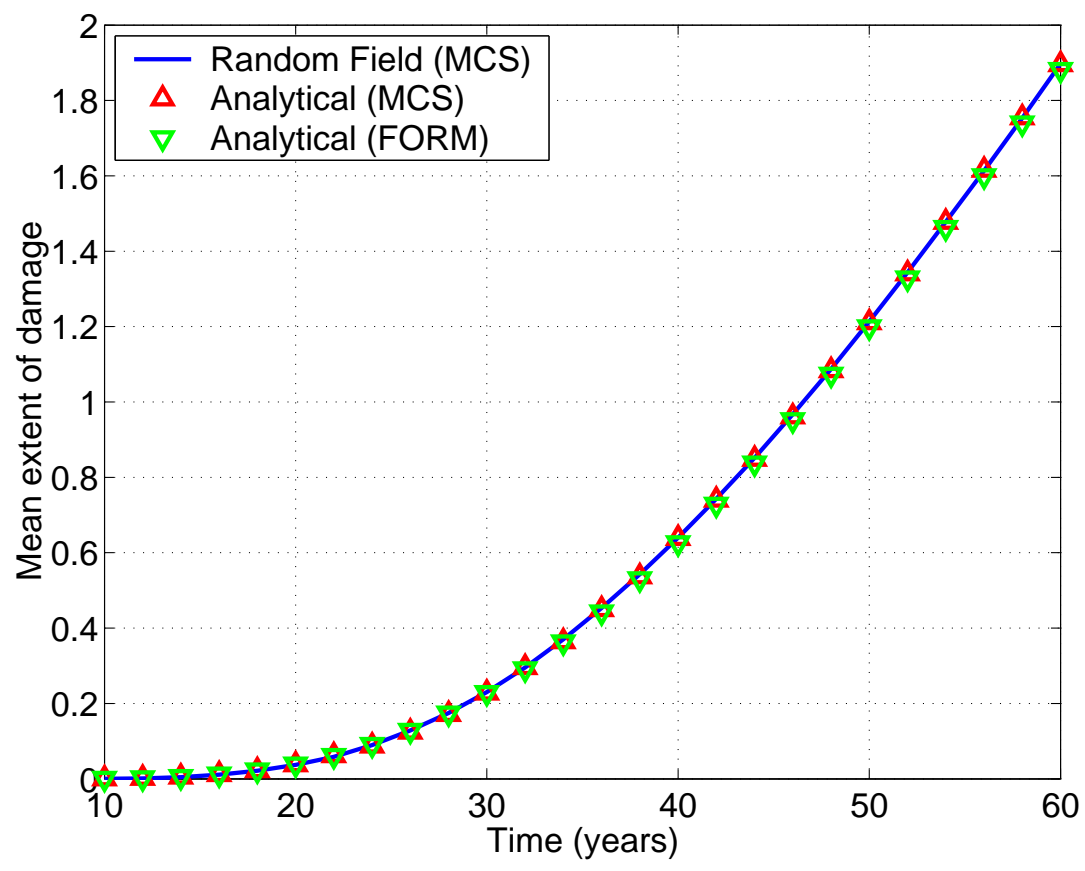

Figure 5: Evolution in time of the mean extent of damage

The mean damage length obtained by the three approaches is plotted in Figure 5 (the values are also gathered in Table 2, columns \#2-4). An excellent agreement between the various approaches is observed. The maximal discrepancy between FORM and the field discretization approach is less than $5 \%$, and less than $2 \%$ for $t \geq 40$ years. Part of this discrepancy is due to the linearization in FORM: 
indeed, if MCS is used for evaluating the point-in-space probability of failure instead of FORM, the latter discrepancy reduces to $3 \%$, and less than $0.5 \%$ for $t \geq 40$ years.

\begin{tabular}{|c|c|c|c|c|c|c|}
\hline \multirow{3}{*}{$\begin{array}{c}\text { Time } \\
\text { (years) }\end{array}$} & \multicolumn{3}{|c|}{ Mean value } & \multicolumn{3}{|c|}{ Standard deviation } \\
\hline & Eq.(11) & Eq.(11) & Random field & Eq.(16) & Eq.(16) & Random field \\
\hline & MCS & FORM & MCS & MCS & FORM & MCS \\
\hline 20 & 0.038 & 0.039 & 0.037 & 0.239 & 0.245 & 0.224 \\
\hline 22 & 0.062 & 0.061 & 0.058 & 0.310 & 0.321 & 0.294 \\
\hline 24 & 0.088 & 0.091 & 0.089 & 0.376 & 0.406 & 0.382 \\
\hline 26 & 0.125 & 0.128 & 0.129 & 0.479 & 0.498 & 0.482 \\
\hline 28 & 0.171 & 0.174 & 0.175 & 0.567 & 0.597 & 0.582 \\
\hline 30 & 0.228 & 0.228 & 0.230 & 0.677 & 0.701 & 0.686 \\
\hline 32 & 0.296 & 0.291 & 0.295 & 0.781 & 0.810 & 0.799 \\
\hline 34 & 0.365 & 0.363 & 0.370 & 0.902 & 0.921 & 0.914 \\
\hline 36 & 0.450 & 0.442 & 0.454 & 1.016 & 1.035 & 1.034 \\
\hline 38 & 0.537 & 0.530 & 0.543 & 1.134 & 1.149 & 1.151 \\
\hline 40 & 0.638 & 0.626 & 0.640 & 1.245 & 1.264 & 1.265 \\
\hline 42 & 0.740 & 0.728 & 0.743 & 1.350 & 1.377 & 1.379 \\
\hline 44 & 0.849 & 0.837 & 0.852 & 1.456 & 1.489 & 1.492 \\
\hline 46 & 0.961 & 0.952 & 0.967 & 1.578 & 1.598 & 1.599 \\
\hline 48 & 1.084 & 1.073 & 1.088 & 1.685 & 1.705 & 1.705 \\
\hline 50 & 1.211 & 1.199 & 1.214 & 1.781 & 1.809 & 1.808 \\
\hline 52 & 1.341 & 1.329 & 1.344 & 1.883 & 1.909 & 1.906 \\
\hline 54 & 1.478 & 1.462 & 1.478 & 1.972 & 2.005 & 2.003 \\
\hline 56 & 1.616 & 1.600 & 1.614 & 2.066 & 2.097 & 2.093 \\
\hline 58 & 1.755 & 1.739 & 1.753 & 2.155 & 2.184 & 2.181 \\
\hline 60 & 1.896 & 1.882 & 1.898 & 2.251 & 2.267 & 2.264 \\
\hline
\end{tabular}

Table 2: Mean and standard deviation of the extent of damage - comparaison of approaches

The standard deviation of the extent of damage is computed by three approaches as well:

- Eq.(16) where the system reliability problem under the integral is solved by FORM;

- Eq.(16) where this problem is solved by MCS (100,000 samples were used);

- the field discretization approach.

The standard deviation of the extent of damage obtained by the three approaches is plotted in Figure 6 (the values are also gathered in Table 2, columns \#5-7). Here again, the agreement between the various approaches is excellent. The maximal discrepancy between the analytical and the field discretization approaches is less than $9 \%$, and about $0.2 \%$ for $t \geq 40$ years. The accuracy of the analytical formula (Eq.(16)) increases with larger time horizons. The use of Monte Carlo simulation for evaluating the system probability of failure (instead of FORM) does not help much. Indeed, part of the residual error is due to the approximation in the quadrature of the integral (16).

The various algorithms to compute the mean and standard deviation of the extent of damage are implemented in MathCad. In terms of efficiency, the field discretization approach requires about 5 hours on a standard PC (Pentium M processor at $1.6 \mathrm{GHz}, 512 \mathrm{MB}$ RAM) to get the curves in Figures 5-6, whereas the analytical approach (FORM and MCS) requires less than 2 minutes. 


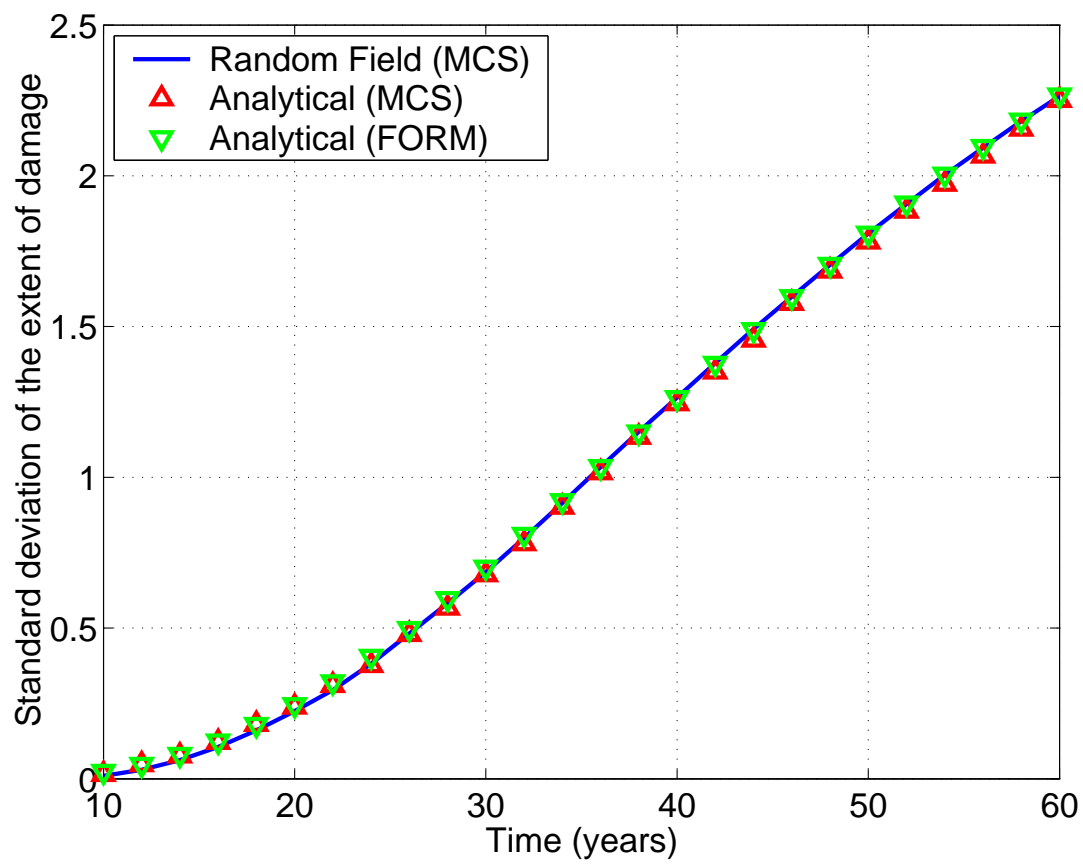

Figure 6: Evolution in time of the standard deviation of the extent of damage

\subsection{Histogram of the extent of damage}

From the Monte Carlo simulations, it is possible to plot histograms of the extent of damage, see Figure 7. It is first observed that there is always a non zero number of spatial realizations of the limit state function that are strictly positive, meaning that the associated realization of the damage length $\mathfrak{e}([0, L], t)$ is exactly equal to zero. In other words, there is a probability spike on zero, as shown in Figure 7. This spike is all the greater since the time instant is smaller (the spikes in the figure are not on scale). Similarily, when time increases (e.g. $t=60$ years), another spike appears for $\mathfrak{e}([0, L], t)=L$. This represents cases where the beam is fully damaged.

It clearly appears in Figure 7 that the damage length has a complex PDF. Thus its approximation by a Gaussian PDF (as e.g. in [6]), due to the application of the central limit theorem on the summation of independent damaged areas, is probably very rough. This is due to the fact that:

- it is impossible to discretize the structure into really independent subdomains, as soon some input parameters are modelled by random variables (i.e. spatially perfectly correlated);

- the extent of damage is by definition bounded by 0 and $|\mathcal{D}|$.

\subsection{Probability of no corrosion}

The probability of having the beam completely sound at a given time instant $(\mathbb{P}(\mathcal{E}([0, L], t)=0))$ is an interesting information to assess the state of damage in time. In the case of uni-dimensional problems $(d=1)$ as the current example, a closed-form expression for this quantity can be derived. 
10 years

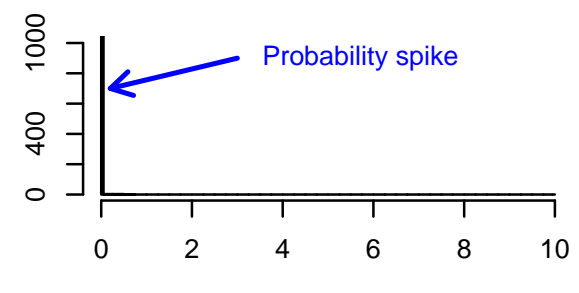

$\mathrm{m}$

30 years

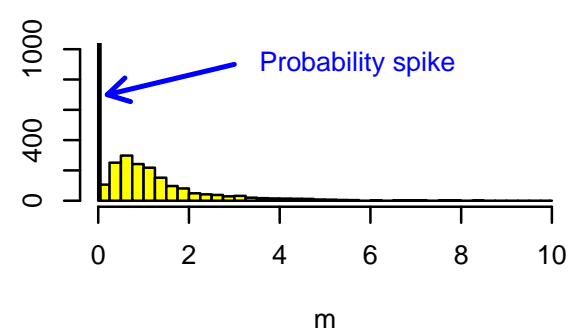

50 years

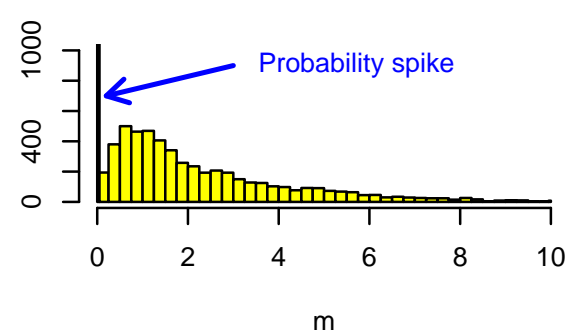

20 years

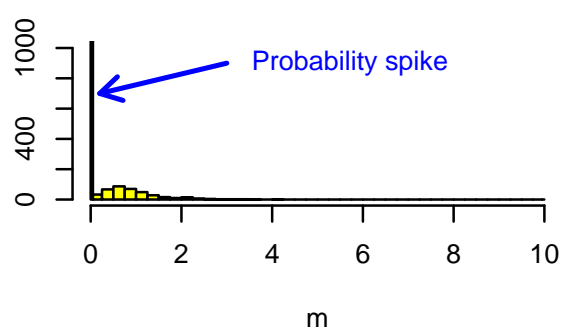

40 years

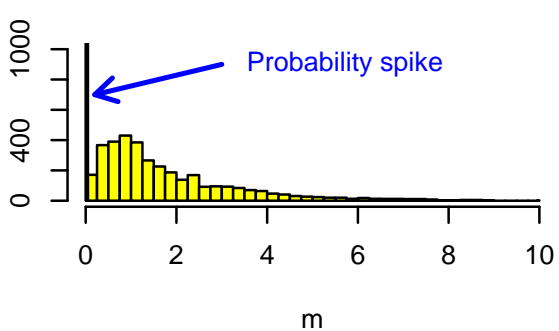

60 years

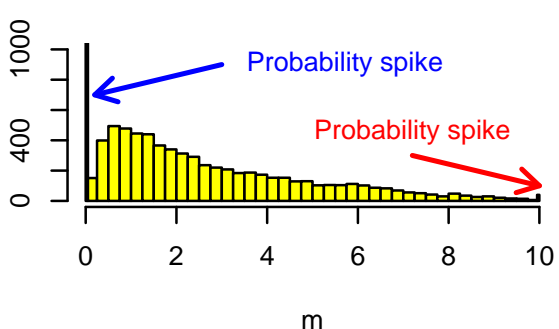

$\mathrm{m}$

Figure 7: Histogram of the extent of damage $(\mathrm{m})$ at various time instants (10,000 samples)

Indeed:

$$
\begin{aligned}
\mathbb{P}(\mathcal{E}([0, L], t)=0) & =1-\mathbb{P}(\mathcal{E}([0, L], t)>0)=1-\mathbb{P}(\exists x \in[0, L], g(\boldsymbol{Z}(x), t) \leq 0) \\
& =1-\mathbb{P}\left(\bigcup_{x \in[0, L]} g(\boldsymbol{Z}(x), t) \leq 0\right)
\end{aligned}
$$

where the righthand side is nothing but the space-variant probability of failure defined in Eq.(7).

This space-variant problem can be considered as a spatial first passage problem, and thus solved by techniques developed in time-variant reliability analysis [11]. The spatial outcrossing rate from the undamaged state to the damage state is defined at each time instant by:

$$
\nu^{+}(x, t)=\lim _{h \longrightarrow 0} \mathbb{P}(g(\boldsymbol{Z}(x), t)>0 \cap g(\boldsymbol{Z}(x+h), t) \leq 0) / h
$$

In case of homogeneous problems, this quantity is independent of $x$. If the occurrence of spatial outcrossing is considered as a Poisson process [14, Chap. 6], the space-variant probability of failure 
may be estimated in the homogeneous case by:

$$
\mathbb{P}(\mathcal{E}([0, L], t)>0) \approx 1-e^{-\nu^{+}\left(x_{0}, t\right)}
$$

where the outcrossing rate is computed at any point $x_{0} \in[0, L]$ due to homogeneity. Hence the probability of no corrosion:

$$
\mathbb{P}(\mathcal{E}([0, L], t)=0) \approx e^{-\nu^{+}\left(x_{0}, t\right)}
$$

The outcrossing rate may be evaluated by the PHI2 method $[15,28]$ which is based on the solution of the parallel system reliability problem in Eq.(49), see the references for details.

Table 3 gathers the probability of no corrosion at various time instants, computed either by the field discretization approach (fraction of the number of spatial realizations of the limit state function for which no corrosion is observed) or by Eq.(51).

\begin{tabular}{ccc}
\hline Time (years) & MCS & Eq.(51) \\
\hline 20 & 0.960 & 0.956 \\
22 & 0.939 & 0.935 \\
24 & 0.913 & 0.910 \\
26 & 0.886 & 0.880 \\
28 & 0.857 & 0.847 \\
30 & 0.823 & 0.812 \\
32 & 0.787 & 0.774 \\
34 & 0.750 & 0.736 \\
36 & 0.715 & 0.698 \\
38 & 0.681 & 0.660 \\
40 & 0.642 & 0.623 \\
42 & 0.607 & 0.587 \\
44 & 0.572 & 0.553 \\
46 & 0.535 & 0.522 \\
48 & 0.502 & 0.492 \\
50 & 0.470 & 0.464 \\
52 & 0.437 & 0.438 \\
54 & 0.409 & 0.414 \\
56 & 0.382 & 0.393 \\
58 & 0.355 & 0.373 \\
60 & 0.330 & 0.355 \\
\hline
\end{tabular}

Table 3: Probability of no corrosion in the beam $(\mathbb{P}(\mathcal{E}([0, L], t)=0))$

It appears that the analytical approximate formula provides an accurate estimation of the probability of having no corrosion, the discrepancy compared to the MCS results being less than $6 \%$. However, it is expected that the accuracy will decrease when time increases, due to the fact that the Poissonian assumption on the outcrossing becomes incorrect when many (possibly correlated) outcrossings occur.

\subsection{Influence of the autocorrelation coefficient function}

As mentioned above, the autocorrelation coefficient function for the input random fields is not well known due to lack of data. In this section, the influence of the shape of the autocorrelation coefficient 
function $\rho_{D}\left(x_{1}, x_{2}\right)$ is studied, as well as that of the scale of fluctuation. The description of the random field modelling the concrete cover is the same as in Table 1. As shown in Section 3.2, changing $\rho_{D}\left(x_{1}, x_{2}\right)$ will not change the expected extent of damage, but rather influence the variance of the latter.

As the analytical approach described above allows to compute the standard deviation of the extent of damage in a matter of seconds, it is possible to use it for carrying out a parametric study. As an illustration, the standard deviation of the extent of damage at $t=60$ years is considered here. The evolution of this quantity as a function of the scale of fluctuation of the coefficient of diffusion $\mathrm{D}_{\mathrm{CO}_{2}}$ is plotted in Figure 8 for two shapes of autocorrelation coefficient functions (Type A, B), see Eqs.(29)-(30)).

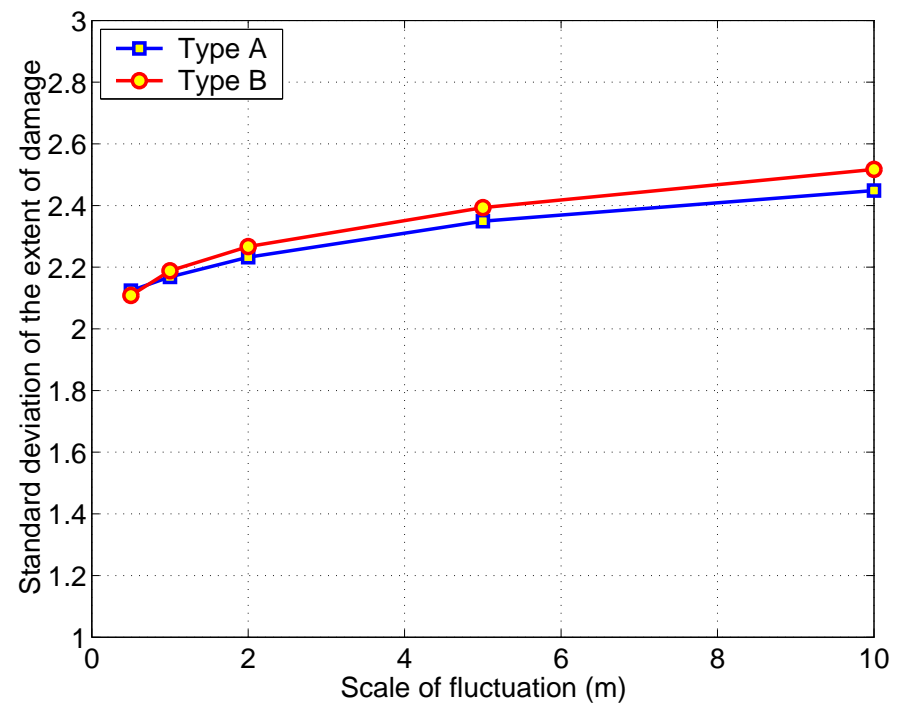

Figure 8: Influence of the shape of the autocorrelation coefficient function and the scale of fluctuation of $\mathrm{DCO}_{2}$

From this figure, it appears that the standard deviation is rather insensitive to the parameters. Indeed, the value varies between $-6 \%$ to $+11 \%$ with respect to the original scenario presented in Section 5.3 (Type $\mathrm{B}, \theta_{D}=2 \mathrm{~m}$ ). This is an interesting result since the shape and scale parameters of the autocorrelation coefficient functions are difficult to infer in practice due to lack of data. 


\section{Conclusions}

The characterization of the extent of damage in probabilistic degradation models of structures is of major interest, especially for optimizing maintenance policies. This requires describing the parameters of the degradation models as random fields.

The paper presents analytical derivations for the mean and standard deviation of the extent of damage. The former appears independent from the correlation structure of the input fields. Both quantities may be computed by analytical formulæ without any discretization of the random fields.

In order to assess the accuracy of the formulæ , an efficient Monte Carlo-based framework for computing spatial realizations of the extent of damage is proposed, based on the EOLE discretization method. On top of mean and standard deviation, the direct Monte Carlo simulation of the extent of damage also yields histograms of the latter.

All the methods are illustrated on the example of a RC beam submitted to carbonation-induced rebars corrosion. The mean and standard deviation of the extent of damage obtained by the various approaches compare very well with each other. The computational cost of the analytical approach is about two orders of magnitude smaller than that of the direct Monte Carlo simulation.

From the histograms of the extent of damage, it appears that probability spikes corresponding to "fully sound" or "fully damaged" structures exist. The approximation of the extent of damage by a Gaussian random variable is thus proven to be irrelevant in this context. These histograms may be used in the context of reliability analysis, e.g. to compute the probability that the extent of damage is greater than a given value, say $5 \%$ of the original length (or surface) of the structure. The existence of probability spikes shall warn the analyst that classical reliability methods such as FORM/SORM may be inappropriate for solving this problem.

Of particular interest in reliability analysis is the problem of computing the probability of having no damage along the structure $(\mathcal{E}(\mathcal{D}, t)=0)$. A technique for computing directly this quantity is finally proposed in the case of one-dimensional problems (see application example). This technique, inspired by time-variant reliability methods, does not require the discretization of the input random fields either.

Although illustrated on a simple degradation model, the proposed approach is quite general and could be applied efficiently to chloride contaminated structures such as bridge decks.

\section{Acknowledgments}

The author would like to thank Mr G. Defaux and Dr M. Pendola (Phimeca Engineering S.A.) for fruitful discussions on space-variant reliability problems. These discussions were made possible through the joint research agreement between Electricité de France, the French Institute for Advanced Mechanics (IFMA, Pr. Lemaire) and Phimeca Engineering S.A.

\section{A Proof of Eqs.(16),(17)}

One-dimensional case : $\mathcal{D}=[0, L]$

Suppose $f\left(x_{1}, x_{2}\right) \equiv \tilde{f}\left(x_{1}-x_{2}\right)$, where $\tilde{f}(z)$ is an even function of its argument. Assume the integral 
$I_{1}=\int_{0}^{L} \int_{0}^{L} f\left(x_{1}, x_{2}\right) d x_{1} d x_{2}$ exists and is to be computed. The following mapping is used:

$$
\begin{aligned}
u & =\frac{x_{1}+x_{2}}{L}-1 \\
v & =\frac{x_{2}-x_{1}}{L}
\end{aligned}
$$

The integral rewrites:

$$
I_{1}=\frac{L^{2}}{2} \int_{-1}^{1} \int_{-a(v) / 2}^{a(v) / 2} \tilde{f}(L v) d u d v
$$

Since the integrand does not depend anymore on $u$, the integration with respect to $u$ provides $a(v)$. Moreover, due to the fact that $\tilde{f}$ is even, the integral with respect to $v$ is twice that computed over $[0,1]$. Finally, for $v \geq 0$, it is easy to show that $a(v)=2-2 v$. Thus:

$$
I_{1}=L^{2} \int_{0}^{1} \tilde{f}(L v)(2-2 v) d v
$$

Two-dimensional case : $\mathcal{D}=\left\{(x, y) \in\left[0, L_{1}\right] \times\left[0, L_{2}\right]\right\}$

Suppose $f\left(x_{1}, y_{1}, x_{2}, y_{2}\right) \equiv \tilde{f}\left(x_{1}-x_{2}, y_{1}-y_{2}\right)$, where $\tilde{f}(x, y)$ is an even function of its arguments. The integral to be computed is of the form:

$$
I_{2}=\int_{x_{1}=0}^{L_{1}} \int_{x_{2}=0}^{L_{1}} \int_{y_{1}=0}^{L_{2}} \int_{y_{2}=0}^{L_{2}} \tilde{f}\left(x_{1}-x_{2}, y_{1}-y_{2}\right) d x_{1} d x_{2} d y_{1} d y_{2}
$$

The mapping in Eq.(52) can be applied on each coordinate $(x, y)$. Thus:

$$
I_{2}=L_{1}^{2} L_{2}^{2} \int_{0}^{1} \int_{0}^{1} \tilde{f}\left(L_{1} v_{1}, L_{2} v_{2}\right)\left(2-2 v_{1}\right)\left(2-2 v_{2}\right) d v_{1} d v_{2}
$$

\section{References}

[1] S. Engelund and J. Sorensen. A probabilistic model for chloride-ingress and initiation of corrosion in reinforced concrete structures. Struc. Safe., 20:69-89, 1998.

[2] M. Stewart and D. Rosowsky. Time-dependent reliability of deteriorating reinforced concrete bridge decks. Struc. Safe., 20:91-109, 1998.

[3] D. Val, M. Stewart, and R. Melchers. Effect of reinforcement corrosion on reliability of highway bridges. Eng. Struc., 20:1010-1019, 1998.

[4] A.T. Vu and M. Stewart. Structural reliability of concrete bridges including improved chlorideinduced corrosion models. Struc. Safe., 22:313-333, 2000.

[5] Y. Li, T. Vrouwenvelder, T. Wijnants, and J. Walraven. Spatial variability of concrete deterioration and repair strategies. Struct. Concrete, 5:121-130, 2004.

[6] K.A.T Vu and M.G. Stewart. Predicting the likelihood and extent of reinforced concrete corrosion-induced cracking. J. Struc. Eng., ASCE, 131(11):1681-1689, 2005.

[7] M.S. Darmawan and M. Stewart. Spatial time-dependent reliability analysis of corroding pretensioned prestressed concrete bridge girders. Struc. Safe, xx:xxx, 2006. in press. 
[8] M.G. Stewart. Spatial variability of damage and expected maintenance costs for deteriorating RC structures. Structure and Infrastructure Engineering, 2(2):79-90, 2006.

[9] M.G. Stewart, J.A. Mullard, and B.J. Drake. Utility of spatially variable damage performance indicators for improved safety and maintenance decisions of deteriorating infrastructure. In Proc. 2nd Int. Forum Eng. Dec. Making (IFED), 2006. Lake Louise, Canada.

[10] Y. Li. Effect of spatial variability on maintenance and repair decisions for concrete structures. $\mathrm{PhD}$ thesis, Delft University of Technology, 2004.

[11] A. Der Kiureghian and Y. Zhang. Space-variant finite element reliability analysis. Comp. Meth. Appl. Mech. Eng., 168:173-183, 1999.

[12] B. Sudret, G. Defaux, and M. Pendola. Introducing spatial variability in the lifetime assessment of a concrete beam submitted to rebar's corrosion. In Proc. 2nd Int. Forum Eng. Dec. Making (IFED), 2006. Lake Louise, Canada.

[13] Y.S. Petryna and W.B. Krätzig. Computational framework for long-term reliability analysis of RC structures. Comp. Meth. Appl. Mech. Eng., 194:1619-1639, 2005.

[14] R-E Melchers. Structural reliability analysis and prediction. John Wiley \& Sons, 1999.

[15] C. Andrieu-Renaud, B. Sudret, and M. Lemaire. The PHI2 method : a way to compute timevariant reliability. Rel. Eng. Sys. Safety, 84:75-86, 2004.

[16] H. Koo and A. Der Kiureghian. FORM, SORM and simulation techniques for nonlinear random vibrations. Technical Report $\mathrm{n}^{o}$ UCB/SEMM-2003/01, University of California at Berkeley, 2003. 185 pages.

[17] M. Abramowitz and I. A. Stegun, editors. Handbook of mathematical functions. Dover Publications, Inc., 1970.

[18] C.C Li and A. Der Kiureghian. Optimal discretization of random fields. J. Eng. Mech., 119(6):1136-1154, 1993.

[19] B. Sudret and A. Der Kiureghian. Stochastic finite elements and reliability : A state-of-the-art report. Technical Report $\mathrm{n}^{\circ}$ UCB/SEMM-2000/08, University of California, Berkeley, 2000. 173 pages.

[20] E. Vanmarcke. Random fields : analysis and synthesis. The MIT Press, Cambridge, Massachussets, 1983.

[21] A. Der Kiureghian and J-B Ke. The stochastic finite element method in structural reliability. Prob. Eng. Mech., 3(2):83-91, 1988.

[22] Y. Liu and R.E. Weyers. Modelling the time-to-corrosion cracking in chloride contaminated reinforced concrete structures. ACI Mater. J., 95:675-681, 1998.

[23] Thoft-Christensen P. FEM modelling of the evolution of corrosion cracks in reinforced concrete structures. In M. Maes and L. Huyse, editors, Proc. 11th IFIP WG7.5 Conference on Reliability and Optimization of Structural Systems, Banff, Canada, pages 221-228, 2004. 
[24] K. Bhargava, A.K. Ghosh, Y. Mori, and S. Ramanujam. Model for cover cracking due to rebar corrosion in RC structures. Eng. Struc., 28(8):1093-1109, 2006.

[25] Coll. New approach to durability design - an example for carbonation induced corrosion. Technical Report n²38, Comité Euro-international du béton, 1997.

[26] C. Alonso, A. Andrade, J. Rodriguez, and J.M. Diez. Factors controlling cracking of concrete affected by reinforcement corrosion. Materials and Structures, 31:435-441, 1998.

[27] P. Broomfield. Corrosion of Steel in Concrete. E\&FN Spon, 1997.

[28] B. Sudret. Analytical derivation of the outcrossing rate in time-variant reliability problems. Struc. Infra. Eng., 2006. to appear. 